\title{
Laboratory Transition Probabilities for Studies of Nucleosynthesis of Fe-group Elements
}

\begin{tabular}{|r|l|}
\hline Journal: & Canadian Journal of Physics \\
\hline Manuscript ID & cjp-2016-0689.R1 \\
\hline Danuscript Type: & Article \\
\hline Complete List of Authors: & $\begin{array}{l}\text { Lawler, James; University of Wisconsin } \\
\text { Sneden, Chris; University of Texas } \\
\text { Cowan, John; Univ. of Oklahoma, Homer L. Dodge Dept. of Physics and } \\
\text { Astronomy } \\
\text { Den Hartog, Elizabeth A.; University of Wisconsin } \\
\text { Wood, Michael; Univ. of St. Thomas, Dept. of Physics }\end{array}$ \\
\hline Keyword: & abundances, metal-poor, supernovae, transition, probabilities \\
\hline & \\
\hline
\end{tabular}

SCHOLARONE ${ }^{\text {IM }}$

Manuscripts 


\section{Laboratory Transition Probabilities for Studies of Nucleosynthesis of Fe-group Elements ${ }^{1}$}

J. E. Lawler, C. Sneden, J. J. Cowan, E. A. Den Hartog, and M. P. Wood

PACS: $26.30 . \mathrm{Ef}, 32.70 . \mathrm{Cs}, 32.70 . \mathrm{Fw}, 95.30 . \mathrm{Ky}, 96.60 . \mathrm{Fs}, 97.10 . \mathrm{Cv}, 97.10 . \mathrm{Ex}$,

J. E. Lawler, Dept. of Physics, Univ. of Wisconsin - Madison, 1150 University Ave., Madison, WI 53706 USA; jelawler@wisc.edu

C. Sneden, Dept. of Astronomy and McDonald Observatory, Univ. of Texas, Austin, TX 78712 USA; chris@verdi.as.utexas.edu

J. J. Cowan, Homer L. Dodge Dept. of Physics and Astronomy, Univ. of Oklahoma, Norman, OK 73019 USA ; jjcowan1@ou.edu

E. A. Den Hartog, Dept. of Physics, Univ. of Wisconsin - Madison, 1150 University Ave., Madison, WI 53706 USA; eadenhar@wisc.edu

M. P. Wood, Dept. of Physics, Univ. of St. Thomas, 2215 Summit Ave., St. Paul, MN 55105 USA;

mpwood@stthomas.edu

\footnotetext{
${ }^{1}$ This article is part of a Special Issue on the 12th International Colloquium on Atomic Spectra and Oscillator Strengths for Astrophysical and Laboratory Plasmas held in Sao Paulo, Brazil July 2016.
} 
Abstract: The synthesis of iron (Fe-) group elements was different in the early Galaxy than it is today. Measurements of the relative Fe-group elemental abundances in old metal-poor stars yield information on the Galactic chemical evolution and some information on early supernovae (SNe). Improved laboratory data on transition probabilities is essential to this effort. It is also essential to understand and map the limits of standard photospheric models based on one dimensional and local thermodynamic equilibrium approximations.

Abstract: IN FRENCH HERE. 


\section{Introduction}

Compelling evidence exists that the synthesis of iron (Fe-) group elements in the early Galaxy, and by inference the early Universe, was different than the synthesis at later times [e.g. 1,2]. This evidence is from metal-poor stars. Such stars contain a sample of the interstellar medium (ISM) when they were formed. Metallicity $[\mathrm{Fe} / \mathrm{H}]$ is a logarithmic quantity referenced to the Sun and it is the proxy of choice for non-primordial material in a star ${ }^{2}$, and metal-poor stars from Solar $[\mathrm{Fe} / \mathrm{H}]=0$ to $[\mathrm{Fe} / \mathrm{H}] \leq-6$ are known. Although the exact relation between "look-back" time and $[\mathrm{Fe} / \mathrm{H}]$ is not yet established, $[\mathrm{Fe} / \mathrm{H}]$ correlates with time before the present. Relative Fe-group abundances from past studies show dramatic (factor of 10 or $1 \mathrm{dex}$ ) differences as a function of $[\mathrm{Fe} / \mathrm{H}]$. The trends are only partially explained by the rise of Type 1a supernovae $(\mathrm{SNe})$. A reliable map of the relative Fe-group abundances as a function of $[\mathrm{Fe} / \mathrm{H}]$ yields details of the Galactic chemical evolution and may reveal important new details of early core collapse SNe. The first generation of stars, as studied using numerical simulations, were massive, rapidly rotating, and by definition had only primordial elements [e.g. 3, 4]. Long (> $2 \mathrm{~s}$ ) Gamma Ray Bursts (GRBs) are now thought to be signals from early core collapse (CC) SNe.

There are serious challenges in measuring the relative Fe-group abundances as a function of $[\mathrm{Fe} / \mathrm{H}]$. Clearly a large selection of spectral lines from neutral and ionized Fe-group species with very reliable transition probabilities are needed to study $F, G$, and $K$ stars from Solar $[\mathrm{Fe} / \mathrm{H}]=0$ to $[\mathrm{Fe} / \mathrm{H}]=-6$. At Solar metallicity strong lines connected to low levels of the Fe-group ion are saturated (optically thick at line center) and unreliable if used for abundance determination. Lines of the Fe-group neutral atom are less likely to be saturated because the singly ionized species dominates by a factor of 10 to 100 or more [5]. The switch from neutral to ion lines with decreasing $[\mathrm{Fe} / \mathrm{H}]$ may initially emphasize weak ion lines to avoid saturation and then employ strong lines of the ion for the most metal-poor stars. Thus laboratory data for a wide range of transition probabilities are needed. The currently preferred method for determining atomic transition probabilities or $\log (g f) s$ is to combine absolute radiative lifetimes from laser induced fluorescence (LIF) with relative emission branching fractions (BFs) from a Fourier transform spectrometer (FTS) or other spectrometer with a high resolving power. Although interferometric FTS instruments have many important advantages for BF measurements, they have one important disadvantage for weak lines. Noise in data from a FTS is typically dominated by photon noise from the strong lines because an interferogram, not a spectrum, is recorded. Photon noise from the entire spectrum is evenly distributed throughout the spectrum. This multiplex noise from the smooth redistribution of all Poisson (quantum or photon) noise in an interferogram is an issue when measuring BFs of weak lines. A weak line may "melt" into the multiplex noise before dominant lines from the common upper level are optically thin. Dispersive spectrometers do not have multiplex noise.

The second major challenge in measuring the relative Fe-group abundances as a function of $[\mathrm{Fe} / \mathrm{H}]$ is the possible breakdown of standard photospheric models [e.g. 6]. Such models are typically

\footnotetext{
${ }^{2}$ We use standard stellar spectroscopic abundance notations. For elements $X$ and $Y$, the relative abundances are written $[X / Y]=\log _{10}\left(N_{X} / N_{Y}\right)_{\text {star }}-\log _{10}\left(N_{X} / N_{Y}\right)_{\text {sun }}$. For element $X$, the "absolute" abundance is written log $\varepsilon(X)=$ $\log _{10}\left(N_{X} / N_{H}\right)+12$. Metallicity is defined as $[\mathrm{Fe} / \mathrm{H}]$.
} 
built on one dimensional (1D) and local thermodynamic equilibrium (LTE) approximations. The electron pressure in a photosphere of an F, G, and K star drops as the metallicity drops because most of the free electrons are from metals with low ionization potentials compared to $\mathrm{H}$. Giant stars are favored over dwarf stars because of the need for copious quantities of photons when recording spectra at high resolving power with high signal-to-noise ratios (S/Ns). Giant stars have even lower electron density than dwarf stars at a similar $[\mathrm{Fe} / \mathrm{H}]$ and much lower atomic densities. The LTE approximation is a source of concern for studies of stars with a large range of stellar parameters.

Convection is easily observed in the Sun, and it results in a pattern of hot and cool areas on the surface of the Sun. Distant stars have convection and its effect on abundance measurement is only partly understood [6]. Four numbers are used to characterize distant stars when using standard 1D/LTE photospheric models: photospheric temperature $T_{\text {eff }}$, surface gravity $\log (\mathrm{g})$, metallicity $[\mathrm{Fe} / \mathrm{H}]$, and microturbulance $v_{t}$. Microturbulance captures some of the effects of increased line width from convective motion. Available computational power has grown by many orders of magnitude since the first photospheric models were developed, and thus it is logical to move toward more advanced photospheric models. A 3D or hydrodynamic simulation combined with a non-LTE (NLTE) or rate equation model is now possible. The primary challenge is the lack of accurate rate constants for NLTE models. Inelastic and superelastic collisions of $\mathrm{H}$ and $\mathrm{He}$ atoms with ground and excited metal atoms and ions are very different than electron collisions. The theoretical approach for calculating inelastic and superelastic electron collision cross sections is similar to that of optical cross sections. Heavy particle collisions are quite different and involve molecular potential curve crossings. Barklem is a leader in the computation of better cross sections and rate constants for inelastic and superelastic heavy particle collisions [e.g. 7, 8, 9] to replace the widely used Drawin approximation [10, 11]. Rate constants for certain charge transfer reactions are also known to be important but not yet available with satisfactory accuracy and precision. Reactions such as $\mathrm{H}^{-}+\mathrm{Fe}^{+} \leftrightarrow \mathrm{H}+\mathrm{Fe}^{*}$ are expected to equilibrate highly excited levels of neutral $\mathrm{Fe}^{*}$ with the ground and low metastable levels of $\mathrm{Fe}^{+}$. The cryogenic electrostatic storage ring DESIREE at Stockholm University will provide accurate, absolute cross sections for important charge transfer reactions at realistic thermal interaction energies using merged beams [12]. Reliable rate constants for other important reactions will also be needed. It is exciting that the first 3D/NLTE models are appearing [13]. Further improvements in the key rate constants will justify switching from 1D/LTE photospheric models to 3D/NLTE models.

A complementary empirical approach to investigate potential 3D/NLTE contributions in stellar photospheres is to measure the relative Fe-group abundances as a function of $[\mathrm{Fe} / \mathrm{H}]$. The lead author's lecture at ASOS 12 emphasized this empirical approach. A few spectral lines can be identified as highly reliable lines (HRLS) for each Fe-group ion. These HRLs connect to the ground and low metastable levels of the ion. The ground and low metastable levels (if such metastable levels exist) are the primary population reservoir of Fe-group material in the photospheres of stars of interest. While low and very low populations of excited levels of the ion and any levels of the neutral atom can be pulled out of thermal equilibrium by various effects, the primary population reservoirs will not be significantly out of thermal equilibrium. Of course, HRLs that are sufficiently well isolated in stellar spectra must be identified. Although one might be concerned about Fe-group material "hiding" in the photosphere as 
doubly ionized species, this is quite unlikely because the second ionization potentials of Fe-group elements are all larger than that of $\mathrm{H}$. Charge exchange reactions such as $\mathrm{Fe}^{+2}+\mathrm{H} \rightarrow \mathrm{Fe}^{+}+\mathrm{H}^{+}$will dominate reverse reactions. Although some of the intrinsically strong HRLs already have well known $\log (g f) s$, many of the weak HRLs do not have well known transition probabilities. Clearly the intrinsically strong HRLs with well known $\log (g f) s$ will be most useful for abundance determinations at low $[\mathrm{Fe} / \mathrm{H}]$, while intrinsically weak lines with very small $\log (g f) s$ have potential to be useful at higher and near Solar $[\mathrm{Fe} / \mathrm{H}]$. The identification of relatively unblended HRLs in stellar spectra and their clear significance naturally leads to increased efforts to measure $\log (g f)$ s for such lines.

The multiplex noise of FTS instruments can make it impossible to measure the BF of a weak line by masking the weak line with photon noise from strong lines. The solution to this problem is to switch to a purely dispersive instrument that produces spectra with the photon noise of strong lines localized at their positions. The aberration-corrected $3 \mathrm{~m}$ focal length echelle spectrometer at the Univ. of Wisconsin was developed to provide the resolving power, broad spectral coverage, and UV sensitivity for BF measurements on weak Fe-group HRLs [14]. It covers the $2000 \AA$ to $4000 \AA$ region with only three CCD frames, and achieves a resolving power of 250,000 with a 50 micron diameter entrance pinhole. Figure 7 of Wood and Lawler demonstrates the UV sensitivity of the $3 \mathrm{~m}$ echelle spectrometer [14].

Mapping of the limits of standard 1D/LTE photospheric models requires larger sets of reliable $\log (g f) s$ for neutral and ionized Fe-group species. Anomalous abundance values from lines connected to a specific, e.g. ground, level of an atom with small scatter compared to lines connecting other levels is clearly a NLTE effect if the $\log (g f) s$ and other line data such as isotopic or hyperfine parameters are reliable. Similarly, observations in a particular wavelength region, e.g. the Balmer continuum, might yield anomalous abundance values due to a breakdown in 1D/NLTE models from something like an incomplete treatment of the continuum opacity. The next two sections of this article are a listing with references for recent Fe-group measurements at Lund University in Sweden, occasionally with Imperial College - London (ICL) and/or the National Institute of Standards \& Technology (NIST) groups, and the Univ. of Wisconsin - Madison, again occasionally with the ICL and/or NIST groups. Important measurements and theoretical results from other institutions have appeared in recent years, but sustained experimental efforts on the Fe-group have been made at Lund, Sweden partially in collaboration with the ICL and/or NIST group, and Madison, Wisconsin, again partially in collaboration with ICL and/or NIST group. These experimental efforts will be emphasized.

It is broadly recognized that BF measurements, and not the LIF radiative lifetime measurements used for normalization, are the primary challenge in improving $\log (g f) \mathrm{s}$. The challenge of BF measurements increases for weak and very weak lines that are becoming more important for elemental abundance studies. Some "friendly" competition and occasional redundant measurement of BF sets by an independent team using different instruments with different radiometric calibrations is critically important to understanding and controlling systematic errors and to ensuring that total uncertainty estimates are realistic. 


\section{Lab Astro Results from the Univ. of Lund , ICL, and NIST - Gaithersburg, MD}

The effort at Lund, Sweden is named the FERRUM Project, and it has focused on Fe II to substantial extent. This effort has included some measurements on highly forbidden lines using the CRYRING ion storage ring. Such forbidden line data on magnetic dipole and electric quadrupole transitions are important for the study of nebula but rarely applicable to stellar photospheres. The forbidden line studies using the CRYRING ion storage ring are mentioned below, in part to illustrate the breadth and accomplishments of the FERRUM Project. It is possible that electric dipole forbidden lines might serve as HRLs in the future or that forbidden line techniques developed in the FERRUM Project might be applied to very weak HRLs in the future.

Hartman et al. [15] used $\mathrm{Fe}^{+}$ions in the CRYRING storage ring for $\log (g f)$ measurements on 13 forbidden lines of Fe II from three metastable levels. Hartman et al. [16] subsequently extended this work to forbidden lines of $\mathrm{Ti}$ II using $\mathrm{Ti}^{+}$ions in the metastable $3 \mathrm{~d}^{2}\left({ }^{3} \mathrm{P}\right) 4 \mathrm{~s} \mathrm{~b}^{4} \mathrm{P}_{5 / 2}$ level with a very long lifetime of 23s! Measurements on forbidden lines of Ti II were immediately applied in studies of Eta Carinae by Hartman et al. [17]. Hartman et al. [18] extended earlier work at CRYRING to Sc II, combined the radiative lifetimes of metastable levels with BFs from observations of Eta Carinae, and determined transition probabilities of forbidden lines in Sc II. Gurell et al. [19] expanded the studies of forbidden lines in Fe II at CRYRING. Lifetime measurements on the $3 d^{6}\left({ }^{3} G\right) 4 s a^{4} G_{11 / 2}$ and $3 d^{6}\left({ }^{3} D\right) 4 s \quad b^{4} D_{1 / 2}$ metastable levels of $\mathrm{Fe}^{+}$were combined with $\mathrm{BFs}$ from Eta Carinae and compared to theoretical values. Although these forbidden line measurements are not generally applicable to studies of stellar photospheres, they do illustrate the breadth and high quality of the effort at Lund, Sweden.

Li et al. [20] used LIF on metastable levels of $\mathrm{Fe}^{+}$to excite levels near $7.5 \mathrm{eV}$ excitation energy in $\mathrm{Fe}^{+}$. Iron dominates astrophysical spectra in general and stellar spectra in particular. Various ionization stages of iron moderate energy transport deep within stars. All or nearly all measurements on Fe are eventually important to astrophysics. Sikstrom et al. [21] continued this work to excite $3 d^{6} 4 p$ levels near 7.7eV and measure BFs for 18 lines of Fe II lines between 2350 and $2800 \AA$. Nilsson et al. [22] used multistep LIF to measure radiative lifetimes of Fe ${ }^{+}$levels near $10 \mathrm{eV}$ and a FTS to measure BFs for 29 lines of the Fe II $4 p-4 d$ transition array. These measurements were immediately useful for studies of the chemically peculiar Hg-Mn star Chi Lupi. Li et al. [23] used vacuum ultraviolet (VUV) LIF to measure radiative lifetimes of the $3 d^{5}\left({ }^{6} \mathrm{~S}\right) 4 s 4 p\left({ }^{3} \mathrm{P}\right) \mathrm{y}^{6} \mathrm{P}^{0}$ levels of $\mathrm{Fe}^{+}$. Karlsson et al. [24] reported new measurements on four $3 d^{6} 5 \mathrm{~s}$ levels of $\mathrm{Fe}^{+}$near $10 \mathrm{eV}$ excitation energy and BF measurements on 20 lines of Fe II. These lines play a major role in Lyman alpha pumped fluorescence of Fe II in astrophysical systems. In a collaboration with the Lund group, Pickering of ICL [25] reported new measurements on BFs of lines from the $3 d^{6}\left(a^{3} F\right) 4 p$ subconfiguration of Fe II in the visible to VUV region. Pickering et al. [25] BF measurements on the lines from the $3 d^{5}\left({ }^{6} S\right) 4 s 4 p\left({ }^{3} P\right) y^{6} P^{\circ}$ levels of $\mathrm{Fe}^{+}$included the $1608 \AA$ VUV line important in ISM studies. Nilsson et al. [26] used a combination of published and new radiative lifetimes from LIF measurements with new BF measurements to determine 119 transition probabilities for lines of $\mathrm{Cr}$ II. Gurell et al. [27] extended the work of the FERRUM project on $\mathrm{Cr}$ II to higher upper levels of the $z^{4} H, z^{2} D, y^{4} F$ and $y^{4} G$ terms to yield transition probabilities for an additional 145 lines. Engström et al. [28] further extended the $\mathrm{Cr}$ II studies to include lines from the $3 d^{4}\left({ }^{5} D\right) 5 s e^{6} D$ term near 
$10 \mathrm{eV}$ excitation energy. Most recently Hartman et al. [29] extended the LIF radiative lifetime measurements on ionized Fe to the $3 d^{6}\left({ }^{5} D\right) 4 d e^{6} \mathrm{G}$ term of Fe+ near 10.4 eV excitation energy using 2photon excitation enhanced by a intermediate level. The LIF radiative lifetime measurements were then combined with BFs from FTS measurements to determine $\log (g f) s$ for 14 lines from the $e^{6} \mathrm{G}$ upper levels.

Additional studies on Fe-group species at Lund include work on forbidden lines of Sc II by Lundin et al. [30] and $\mathrm{Cr}$ II by Backstrom et al. [31]. The infrared region was emphasized in some work on Sc I by Pehlivan et al. [32]. Lundberg et al. emphasized high excitation levels of Ti II in some very recent work [33].

Additional studies on Fe-group species at ICL, separate from the Ferrum Project, include work by Blackwell-Whitehead et al. on Ti I and Mn I [34, 35, 36] with some emphasis on the infrared. The above mentioned work on Fe II by Pickering et al. [25] was further extended [37]. Ruffoni et al. reported new infrared results on Fe I [38]. NIST scientists participated in [34, 35] and in some work on Mn II [39, 40].

\section{Lab Astro Results from the Univ. of Wisconsin - Madison, ICL, and NIST}

The UW-Madison lab astro effort contributed work on Fe I and Fe II decades ago. The O'Brian et al. work [41] on Fe I and Bergeson et al. work [42] on Fe II is representative of early activities using the combination of LIF radiative lifetimes and BF measurements from FTS data. Large sets of radiative lifetimes from LIF measurements on neutral and ionized Fe-group species were also measured for subsequent combination with BF measurements to determine $\log (g f) s$ [e.g. 43, 44, 45]. Additional activities on VUV lines of Fe II, Co II, Ni II, and other spectra in the mid 1990's were motivated in part by studies of the ISM using the newly launched Hubble Space Telescope [e.g. 46, 47, 48].

The "return to the Fe-group", motivated by studies of metal-poor stars, started with work using FTS data on Cr I [49]. The measurement and application of BFs for 263 lines of $\mathrm{Cr}$ I, in combination with radiative lifetimes from earlier LIF measurements, revealed departures from Saha balance for $\mathrm{Cr}$ in metal-poor stars, and most surprisingly in the Sun. Except for resonance lines of $\mathrm{Cr}$ l, the Saha balance problem has been mitigated by new $\mathrm{Cr}$ II measurements discussed later in this section. Den Hartog et al. [50] reported new LIF radiative lifetime and FTS BF measurements that were combined to generate a set of $\log (g f) s$ for 15 lines of $\mathrm{Mn} \mathrm{II} \mathrm{and} 47$ lines of $\mathrm{Mn}$ I with exceptionally small uncertainties in the \pm 0.01 dex range. The unusual structure of $\mathrm{Mn} \mathrm{I}$ and $\mathrm{Mn} \mathrm{II}$, with lines clustered in very tight and pure RussellSaunders multiplets, enabled us to achieve exceptional accuracy on BFs of Mn I and Mn II. Such small uncertainties are not generally possible or even needed for most spectra. Applications of these new data, which are highly reliable for resonance lines of $\mathrm{Mn} \mathrm{I}$, revealed departures from LTE that affected the ground level of neutral $\mathrm{Mn}$ in metal-poor dwarf and giant stars. The decommissioning of the $1 \mathrm{~m}$ FTS at the National Solar Observatory (NSO) as well as the realization of the importance of intrinsically weak lines led to the increasing dependence on the $3 \mathrm{~m}$ echelle spectrometer in combination with archived FTS data from the NSO. Lawler et al. [51] reported new BF measurements on Ti I from a combination of FTS and echelle data that provided $\log (g f) \mathrm{s}$ for 948 lines normalized using radiative lifetimes from LIF. The lead observer, Chris Sneden, and lead nuclear modeler, John Cowan, of our 
collaboration selected the Sun and metal-poor dwarf star HD 84937 as test stars for new data from this program. The metal-poor dwarf star HD 84937 provides an opportunity to see NLTE effects without running a risk of being overwhelmed by such effects. Furthermore, many high resolution spectra with high $\mathrm{S} / \mathrm{N}$ over large wavelength ranges are available for this star. Finally, parallax measurements on HD 84937 reduce uncertainties in stellar parameters.

Wood et al. [52] reported new BF measurements on Ti II that yielded log(gf)s for 347 lines normalized using radiative lifetimes from LIF. The applicability of a 1D/LTE photospheric model to HD 84937 is strongly supported by comparisons of abundance results from lines of Ti I and Ti II. Wood et al. [53] subsequently used the approach of combining FTS and $3 \mathrm{~m}$ echelle data to work on Ni I, yielding new BF measurements and $\log (g f) s$ for 371 lines. Unfortunately, there are no useful lines of $\mathrm{Ni} I \mathrm{II}$ the optical for a Saha test on this element. The $\log (g f)$ values of UV lines of $\mathrm{Ni}$ II are fairly well known from earlier work [e.g. 48]. In collaboration with the FTS group at IC-London, new radiative lifetime measurements from LIF on highly excited levels of neutral Fe were combined with BF measurements using the FTS at IC-London by Ruffoni et al. [54]. New $\log (g f)$ measurements for a total of 142 lines of Fe I were included in this study that was motivated in part by the Gaia mission of ESO. Den Hartog et al. continued work on Fe I and added $\log (g f) s$ for an additional 203 lines [55]. The next set of BF measurements from a combination of FTS and $3 \mathrm{~m}$ echelle spectra by Wood et al. [56] was 203 lines of V II with new LIF radiative lifetime measurements by Den Hartog et al. [57]. This was quickly followed with new BF measurements by Lawler et al. [58] for 836 lines of V I. The resulting $\log (g f)$ s for V I confirmed a good Saha balance for $V$ in the Sun and HD 84937 [58]. A recent challenge by Holmes et al. [59] to the BFs and $\log (g f) s$ for six of the 836 lines in Lawler et al. [58] is under review. The tentative conclusions of the review confirmed the accuracy of Ar I branching ratios [60] used for calibration of FTS data and support the accuracy of the $\log (g f) s$ in Lawler et al. [58]. The most recent published work based on a combination of FTS and 3m echelle data is that by Lawler et al. [61] on lines of Co I normalized with LIF radiative lifetimes from Nitz et al. [62]. It includes new BF measurements and log(gf)s for 898 lines of Co I. A set of measurements on deep UV lines of Co II is in progress. Lastly, a set of new BF measurements on 183 lines of $\mathrm{Cr}$ II from a combination of FTS and $3 \mathrm{~m}$ echelle data has been submitted for publication [63]. A small number of new LIF radiative lifetime measurements are also included. These measurements test and refine the earlier measurements by Nillson et al. [26] on lines of $\mathrm{Cr}$ II.

\section{Astrophysical Results from HD 84937, Saha Balance, Nuclear Physics}

It is anticipated that NLTE effects appear in all stellar spectra at some level, thus the limits of 1D/LTE models need to be mapped for exploration of Fe-group nucleosynthesis in the early Galaxy. As the metallicity $[\mathrm{Fe} / \mathrm{H}]$ and surface gravity $\log (\mathrm{g})$ of a star both decrease, it is expected that NLTE effects will become more significant. It is essential to determine the affected energy levels or transitions, wavelength regions, and magnitude of such effects. If the NLTE effects in an abundance determination are order 0.01 dex, they are masked by many other effects in stellar spectral analysis including line blending and continuum placement. If the NLTE effects are order 0.1 dex then they are of some concern. 
The selection of the metal-poor turn-off star HD 84937 as a test case for new $\log (g f)$ measurements was based on several attractive qualities: (a) the excellence of photometric data and a high-precision parallax; (b) the quantity of available high-resolution, high $\mathrm{S} / \mathrm{N}$ spectra spanning the wavelength range 2300-9000 $\AA$; and (c) the ability to derive accurate $T_{\text {eff }}$ and $\log (g)$ values without the use of spectroscopic data. One expects that this metal-poor $([\mathrm{Fe} / \mathrm{H}]=-2.32)$ main-sequence turn-off star would have only modest NLTE effects and thus a 1D/LTE photospheric model would yield generally consistent results. This expectation has been fulfilled. Sneden et al. [5] used synthetic spectrum computations of about 1150 transitions of the 10 Fe-group elements to show that the first and second spectra yield the same abundances in HD 84937. Here we summarize their results.

In Figure 1 we plot individual line abundances for eight Fe-group elements in HD 84937 as functions of line wavelength. $\mathrm{Cu}$ and $\mathrm{Zn}$ are not included in this figure because they show only a few neutral-species lines in this star, and have older transition data. Analyses of $\mathrm{Sc}, \mathrm{Cr}, \mathrm{Mn}$, and Fe are discussed in detail in [5], with more detailed plots of their abundances in that paper's Figures 3-6. Abundances of Ti are from [51, 52], $V$ from [56, 57], Co from [61], and Ni from [53]. In total seven Fegroup elements have both first and second spectra transitions in HD 84937. The line-to-line scatters for individual species are generally very small, with sample standard deviations almost always $<0.09$ dex. The original papers cited here should be consulted for detailed discussions of each species.

In Figure 2 we plot the mean Fe-group abundances of HD 84937. Neglecting Cu and Zn, all of the species studied in detail have abundances based on at least eight lines; most species are represented by more than 20 lines. The elements Ti-Ni have both first and second spectra transitions, and the mean abundances from those species are always in agreement to within the small individual uncertainties. Therefore LTE Saha ionization equilibrium appears to hold in the atmosphere of HD 84937 - the derived relative abundances reflect real nucleosynthetic effects, not atmospheric effects.

There is a significant caveat to the happy concordance between the first and second spectra of the Fe-group elements. In order to achieve satisfactory ionization balance for Mn in HD 84937 it is necessary to ignore the abundances derived from the resonance lines of $\mathrm{Mn}$ I at 4030.8, 4033.1, 4034.5 $\AA$ that connect to the ground levels of this species. The resonance lines, marked with $\times$ symbols in Figure 1 , yield abundances that are systematically smaller than those derived from higher-excitation $\mathrm{Mn} I$ and all Mn II lines; see $\$ 3.5$ of [5] and its Figure 6. Similar precautions apply to the ground-state resonance lines of $\mathrm{Cr}$ I at 3578.7, 3593.5, 3605.3, 4254.3, 4274.8, 4289.7 Å. In [63] new transition lab data for Cr II are presented and the $\mathrm{Cr}$ I resonance line issue is discussed; the resulting abundances are closer to ionization equilibrium than seen in Figure 2. The $\mathrm{Mn} \mathrm{I}$ and $\mathrm{Cr} I$ resonance lines have highly reliable $\log (g f)$ values because they have BFs very close to unity and multiple independent LIF lifetime measurements. This is clear evidence that the ground level of both neutral $\mathrm{Cr}$ and $\mathrm{Mn}$ are falling out of LTE. Excited levels of neutral $\mathrm{Cr}$ and $\mathrm{Mn}$ are in LTE to within the scatter, typically less than \pm 0.07 dex, of abundance determinations.

Unexpected and at this time unexplained abundance deviations at the $\sim 0.3$ dex level have also appeared in the Balmer continuum wavelength region. Roederer et al. [64] observed the "Balmer dip" in the wavelength region below $3645 \AA$ using Fe I lines during studies of four metal-poor stars. Roederer 
et al. tested various approximations for terminating the Balmer series and used a selected set of Fe I lines thought to have the most reliable $\log (g f)$ values in unsuccessful attempts to explain the Balmer dip. The Balmer dip reappeared again during work on Ti II by Wood et al. [52]. In the case of Ti II there are many lines in the wavelength region of the Balmer dip and this enabled us to make additional cuts through our data. Curiously, the Balmer dip did not appear using lines with low excitation potential (E.P. or lower level energy) and was clearly visible for lines with E.P. $>1.25 \mathrm{eV}$. This observation is important for two reasons. The first is that the Ti II lines with E.P. $>1.25 \mathrm{eV}$ are stronger, easier to measure branches which in many cases have redundant BF measurements by Pickering et al. [65] and Wood et al. [52]. The second reason is that the E.P. dependence may be a clue that both NLTE and 3D effects are somehow involved.

The data of Figure 2 confirm that relative Fe-group abundance values are significantly non-Solar at a metallicity $[\mathrm{Fe} / \mathrm{H}]=-2.32$. In addition to the well-known relative $\mathrm{Mn}$ deficiency shown by almost all metal-poor stars, there is a marked overabundance of the Sc-Ti-V trio at the light end of the Fe-group elements. In [5] it is argued that correlated variations from star-to-star of [Sc, Ti, V/Fe] can be found in the literature; this phenomenon has not received much attention to date, but deserves additional scrutiny. 


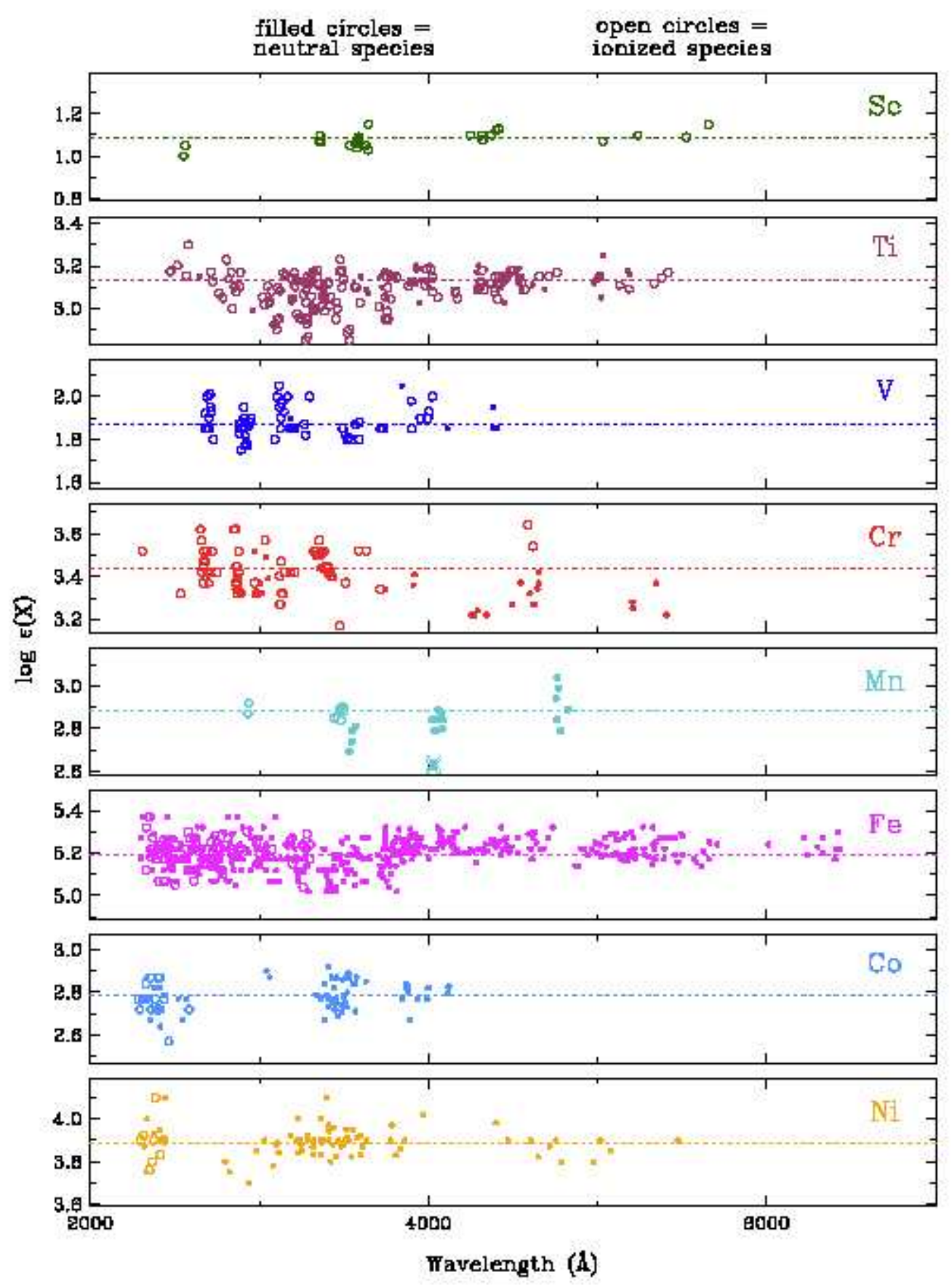

Figure 1. Elemental abundances from first and second spectra lines of eight Fe-group elements in HD 84937. This figure is constructed with abundances of Sc, $\mathrm{Cr}, \mathrm{Mn}$, and Fe presented in [5] (see its Figures 3-6), while those for $\mathrm{Ti}, \mathrm{V}, \mathrm{Co}$, and $\mathrm{Ni}$ are from earlier papers in the same series; summaries and commentary on these abundances are given in [5]. Cu and $\mathrm{Zn}$ are not shown here because they display only a handful of neutral-species lines that have not been investigated by the Wisconsin atomic physics group. 


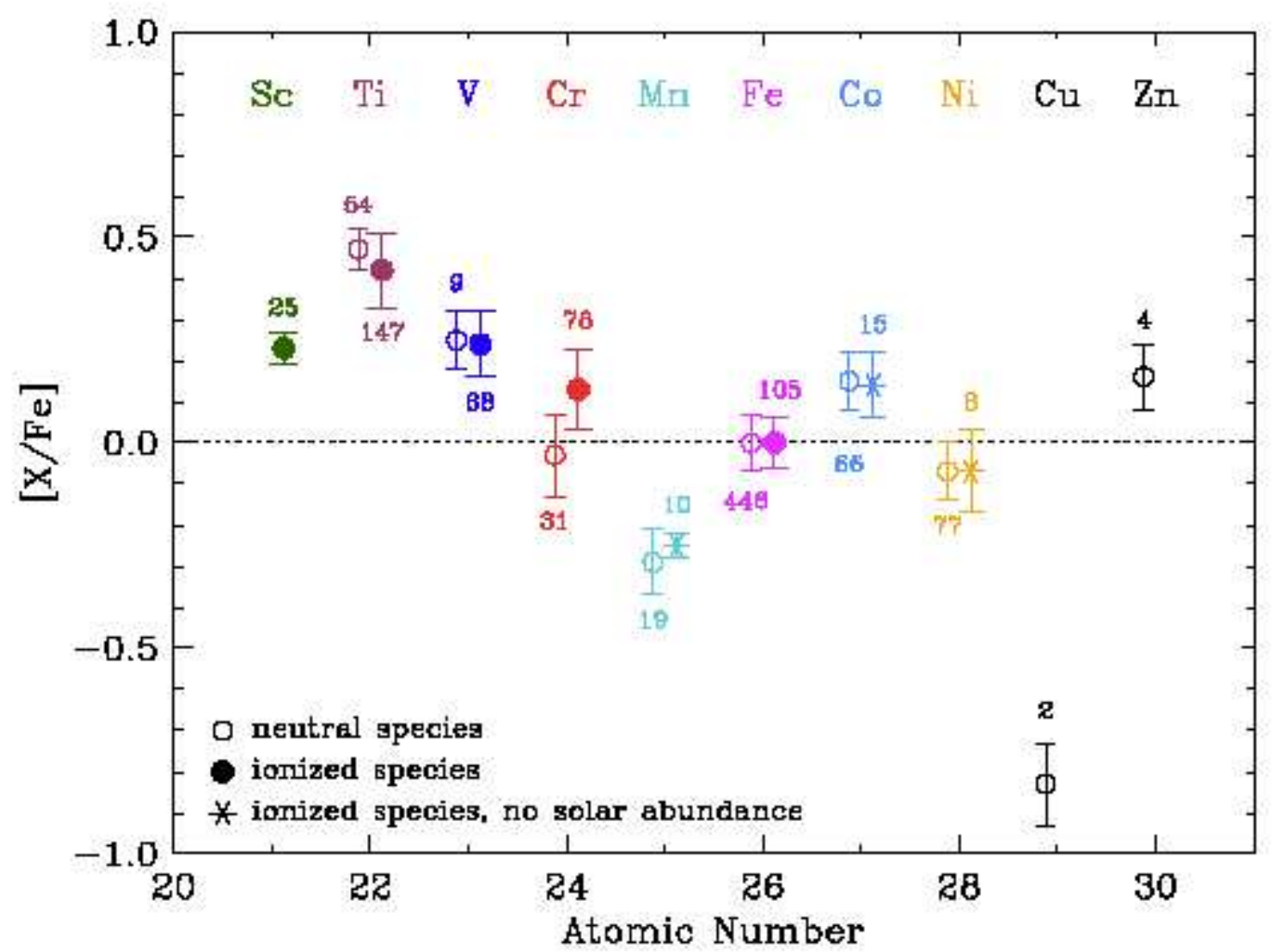

Figure 2. Abundance of Fe-group elements from first and second spectra lines normalized to that of Fe with the Sun as a reference. The error bars are $\pm \sigma$, the sample standard deviations. The number written above or below a species abundance is the number of individual transitions contributing to the mean value shown in this figure. $\mathrm{Cu}$ and $\mathrm{Zn}$ abundances are entered here, but are based on just a few lines of only their neutral species.

As discussed above the chemical abundances in halo stars are "fossils" or "relics" of nucleosynthesis from the first generation(s) of stars in our Galaxy. Those early stars were likely massive and have long since disappeared, while the low-mass (now observed) metal-poor halo stars have extremely long lifetimes. The early synthesis of Fe-peak elements (from scandium to zinc, Z=21-30) was primarily in core-collapse (CC) SNe. In such events a massive star collapses under gravitational pressure as nuclear fuel sources are exhausted. The Fe-peak elements are produced in the Si fusion (or "burning") shell surrounding the collapsing Fe core. Very high temperatures (sometimes in excess of 5 billion degrees) and densities occur in this synthesis $[66,67]$, thus the fusion normally takes place during the actual explosion of the outer part of the star. These Fe-peak elements, along with other elements, are explosively ejected into the ISM and incorporated into gas that will eventually form new stars, 
including the generation of the halo stars. (The inner core of the SNe collapses and becomes a nascent neutron star or black hole.) Fe-peak nuclei including ${ }^{56} \mathrm{Fe}$ and others are mostly produced by a series of alpha (helium nuclei) captures on ${ }^{28} \mathrm{Si}$ or ${ }^{32} \mathrm{~S}$ [67]. Although the merging of two ${ }^{28} \mathrm{Si}$ nuclei might seem more direct, the reaction is inhibited by nuclear physics - particularly the large Coulomb barriers. Instead, where the temperature is very high, elements such as $\mathrm{Fe}, \mathrm{Ni}$, and $\mathrm{Co}$ are formed as a result of complete silicon burning; while in regions that do not experience such high temperatures, other elements such as $\mathrm{Cr}$ and $\mathrm{Mn}$ are formed via incomplete Si burning with only partial Si exhaustion [66]. Understanding the details of these processes, occurring at enormously high temperatures and densities, is difficult because the nuclear environments are not reproducible in the lab. In addition the stellar evolution of stars that become CC SNe is quite complicated. Thus, the accurate abundances of these Fepeak elements, based upon improved and precise lab atomic data, detected in the metal-poor halo stars provides a direct measurement (or a probe) of the conditions in the CC SNe explosion and the Fe-group nucleosynthesis [5].

One intriguing aspect of our recent work has been the indication of correlations among certain Fe-peak elements. Specifically, we find that V, Ti and Sc are correlated and likely produced in the same, or a similar, astrophysical and nuclear environment [5,63]. Our most recent work [63] also shows a correlation among these elements and $\mathrm{Cr}$. We confirmed that $\mathrm{Ca}$, the element below $\mathrm{Sc}$, and $\mathrm{Mn}$, the element above $\mathrm{Cr}$, do not appear to be correlated with the production of the Fe-peak elements Sc, V, Ti, and $\mathrm{Cr}$. Again, this result - based upon precise atomic lab data and high resolution spectral observations - provides new clues about the actual synthesis process of these elements that occurred in SNe billions of years ago during the early history of our Galaxy.

\section{Conclusions}

Spectroscopic data for first and second spectra lines of Fe-group elements are being improved by vigorous lab astro efforts at several institutions. An advanced technique for atomic transition probability measurements based on a combination of radiative lifetimes from LIF measurements with BFs from high resolution emission spectra is yielding highly reliable absolute $\log (g f) s$. A standard 1D/LTE photospheric model is satisfactory for the analysis of Fe-group abundances in the metal-poor dwarf star HD 84937. The only breakdowns of 1D/LTE models identified thus far are confined to the ground levels of neutral $\mathrm{Mn}$ and $\mathrm{Cr}$ and to the wavelength region of the Balmer continuum. The relative abundances of Fe-group elements in HD 84937 are distinctly non-Solar, and a clustering of abundance values for $\mathrm{Sc}, \mathrm{V}, \mathrm{Ti}$, and $\mathrm{Cr}$ is observed.

\section{ACKNOWLEDGEMENTS}

This work is supported in part by NASA grant NNX16AE96G (J.E.L.), by NSF grant AST-1516182 (J.E.L. and E.D.H.), and NSF grant AST-1211585 (C.S.). J.J.C. acknowledges support by the National Science Foundation under Grant No. PHY-1430152 (JINA Center for the Evolution of the Elements).

\section{References}

1. A. McWilliam, Annu. Rev. Astron. Astrophys. 35, 503 (1997). 
2. R. B. C. Henry, J. J. Cowan, and J. Sobeck, Astrophys. J. 709, 715 (2010).

3. V. Bromm, and R. B. Larson, Annu. Rev. Astron. Astrophys. 42, 79 (2004).

4. S. C. Yoon, and N. Langer, Astron. Astrophys. 443, 643 (2005).

5. C. Sneden, J. J. Cowan, C. Kobayashi, M. Pignatari, J. E. Lawler, E. A. Den Hartog, and M. P. Wood, Astrophys. J. 817: 53 (2016).

6. M. Asplund, Annu. Rev. Astron. Astrophys. 43, 481 (2005).

7. P. S. Barklem, Phys. Rev. A 93: 0042705 (2016).

8. P. S. Barklem and J. Aspelund-Johansson, Astron. Astrophys. 435, 373 (2005).

9. P. S. Barklem, A. K. Belyaev, M. Guitou, N. Feautrier, F. X. Gadéa, and A. Spielfiedel, Astron. Astrophys. 530: A94 (2011).

10. H.-W. Drawin, Z. Phys. 211, 404 (1968).

11. H.-W. Drawin, Z. Phys. 225, 483 (1969).

12. H. T. Schmidt R. D. Thomas, M. Gatchell, S. Rosén, P. Reinhed, P. Löfgren, L. Brännholm, M. Blom, M. Björkhage, E. Bäckström, J. D. Alexander, S. Leontein, D. Hanstorp, H. Zettergren, L. Liljeby, A. Källberg, A. Simonsson, F. Hellberg, S. Mannervik, M. Larsson, W. D. Geppert, K. G. Rensfelt, H. Danared, A. Paál, M. Masuda, P. Halldén, G. Andler, M. H. Stockett, T. Chen, G. Källersjö, J. Weimer, K. Hansen, H. Hartman, and H. Cederquist, Rev. Sci. Instrum. 84: 055115 (2013).

13. A. M. Amarsi, K. Lind. M. Asplund, P. S. Barklem, and R. Collet, Mon. Notices Roy. Astron. Soc. Submitted.

14. M. P. Wood, and J. E. Lawler, Appl. Opt. 51, 8407 (2012).

15. H. Hartman, A. Derkatch, M. P. Donnelly, T. Gull, A. Hibbert, S. Johansson, H. Lundberg, S. Mannervik, L.-O. Norlin, D. Rostohar, P. Royen, and P. Schef , Astron. Astrophys. 397, 1143 (2003).

16. H. Hartman, D. Rostohar, A. Derkatch, P. Lundin, P. Schef, S. Johansson, H. Lundberg, S. Mannervik, L.-O. Norlin, and P. Royen, J. Phys B 36, L197 (2003).

17. H. Hartman, P. Schef, P. Lundin, A. Ellmann, S. Johansson, H. Lundberg, S. Mannervik, L.-O. Norlin, D. Rostohar, and P. Royen, Mon. Not. Roy. Astron. Soc. 361, 206 (2005).

18. H. Hartman, J. Gurell, P. Lindin, P. Schef, A. Hibbert, H. Lundberg, S. Mannervik, L.-O. Norlin, and P. Royen, Astron. Astrophys. 480, 575 (2008).

19. J. Gurell, H. Hartman, R. Blackwell-Whitehead, H. Nilsson, E. Bäckström, L. O. Norlin, P. Royen, and S. Mannervik, Astron. Astrophys. 508, 525 (2009). 
20. Z. S. Li, H. Lundberg, C. M. Sikstrom, and S. Johansson, Eur. Phys. J. D 6, 9 (1999).

21. C. M. Sikstrom, M. Schultz-Johanning, M. Kock, Z.-S. Li, H, Nilsson, S. Johansson, H. Lundberg, and A. J. J. Raassen , J. of Phys B 32, 5687 (1999).

22. H. Nillson, C. M. Sikstrom, Z. S. Li, H. Lundberg, A. J. J. Raassen, S. Johansson, D. S. Leckrone, and S. Svanberg, Astron. Astrophys. 362, 410 (2000).

23. Z. S. Li, H. Lundberg, U. Berzinsh, S. Johansson, and S. Svanberg, J. of Phys B33, 5593 (2000).

24. H. Karlsson, C. M. Sikstrom, S. Johansson, Z. S. Li, and H. Lundberg, Astron. Astrophys. 371, 360 (2001).

25. J. C. Pickering, M. P. Donnelly, H. Nilsson, A. Hibbert, and S. Johansson, Astron. Astrophys. 396, 715 (2002).

26. H. Nilsson, G. Ljung, H. Lundberg, and K. E. Nielsen, Astron. Astrophys. 445, 1165 (2006).

27. J. Gurrell, H. Nillson, L. Engström , H. Lundberg, R. Blackwell-Whitehead, K. E. Nielsen, and S. Mannervik, Astron. Astrophys. 511: A68 (2010).

28. L. Engström, H. Lundberg, H. Nilsson, H. Hartman, and E. Bäckström, Astron. Astrophys. 570: A34 (2014).

29. H. Hartman, H. Nilsson, L. Engström, and H. Lundberg, Astron. Astrophys. 584: A24 (2015).

30. P. Lundin, J. Gurell, S. Mannervik, P. Royen, L. O. Norlin, H. Hartman, A. Hibbert, Physica Scripta 78:015301 (2008).

31. E. Backstrom, J. Gurell, P. Royen, S. Mannervik, L. Norlin, R. Blackwell-Whitehead, H. Hartman, and H. Nilsson, Mon. Not. Roy. Astron. Soc. 420, 1636 (2012).

32. A. Pehlivan, H. Nilsson, and H. Hartman, , Astron. Astrophys. 582:A98 (2015).

33. H. Lundberg, H. Hartman, L. Engstrom, H. Nilsson, A. Persson, P. Palmeri, P. Quinet, V. Fivet, G. Malcheva, and K. Blagoev, Mon. Not. Roy. Astron. Soc. 460, 356 (2016).

34. R. J. Blackwell-Whitehead, H. Lundberg, G. Nave, J. C. Pickering, H. R. A. Jones, Y. Lyubchik, Y. V. Pavlenko, and S. Viti, Mon. Not. Roy. Astron. Soc. 373, 1603 (2006).

35. R. J. Blackwell-Whitehead, H. L. Xu, J. C. Pickering, G. Nave, and H. Lundberg, Mon. Not. Roy. Astron. Soc. 361, 1281 (2005).

36. R. Blackwell-Whitehead and M. Bergeman, Astron. Astrophys. 472: L43 (2007).

37. J. C. Pickering, S. Johansson, and P. L. Smith, Astron. Astrophys. 377, 361 (2001).

38. M. P. Ruffoni, C. Allende Prieto, G. Nave, and J. C. Pickering, Astrophys. J. 779:17 (2013). 
39. R. Kling and U. Griesmann, Astrophys. J. 531, 1173 (2000).

40. R. Kling, R. Schnabel, and U. Griesmann, Astrophys. J. Suppl. Ser. 134, 173 (2001).

41. T. R. O'Brian, M. E. Wickliffe, J. E. Lawler, W. Whaling, and J. W. Brault, J. Opt. Soc. Am. B 8, 1185 (1991).

42. S. D. Bergeson, K. L. Mullman, M. E. Wickliffe, J. E. Lawler, U. Litzen, and S. Johansson, Astrophys. J. 464, 1044 (1996).

43. G. C. Marsden, E. A. Den Hartog, J. E. Lawler, J. T. Dakin, and V. D. Roberts, J. Opt. Soc. Am. B 5, 606 (1988).

44. S. Salih and J. E. Lawler, Astron. \& Astrophys. 239, 407 (1990), AND J. E. Lawler, Astron. Astrophys. 252, 853 (1991).

45. J. C. Cooper, N. D. Gibson, and J. E. Lawler, J. Quant. Spectrosc. Radiat. Transfer 58, 85 (1997).

46. K. L. Mullman, M. Sakai, and J. E. Lawler, Astron. Astrophys. Suppl. Ser. 122, 157 (1997).

47. K. L. Mullman, J. E. Lawler, J. Zsargo, and S. R. Federman, Astrophys. J. 500, 1064 (1998).

48. J. A. Fedchak and J. E. Lawler, Astrophys. J. 523, 734 (1999).

49. J. S. Sobeck, J. E. Lawler, and C. Sneden, Astrophys. J. 667, 1267 (2007).

50. E. A. Den Hartog, J. E. Lawler, J. S. Sobeck, C. Sneden, and J. J. Cowan Astrophys. J. Suppl. Ser. 194: 35 (2011).

51. J. E. Lawler, A. Guzman, M. P. Wood, C. Sneden, and J. J. Cowan, Astrophys. J. Suppl. Ser. 205: 11 (2013).

52. M. P. Wood, J. E. Lawler, C. Sneden, and J. J. Cowan, Astrophys. J. Suppl. Ser. 208: 27 (2013).

53. M. P. Wood, J. E. Lawler, C. Sneden, and J. J. Cowan, Astrophys. J. Suppl. Ser. 211:20 (2014).

54. M. P. Ruffoni, E. A. Den Hartog, J. E. Lawler, N. R. Brewer, K. Lind, G. Nave, and J. C. Pickering, Mon. Not. R. Astron. Soc. 441, 3127 (2014).

55. E. A. Den Hartog, M. P. Ruffoni, J. E. Lawler, J. C. Pickering, K. Lind, and N. R. Brewer, Astrophys. J. Suppl. Ser. 215: 23 (2014).

56. M. P. Wood, J. E. Lawler, E. A. Den Hartog, C. Sneden, and J. J. Cowan, Astrophys. J. Suppl. Ser. 214: 18 (2014).

57. E. A. Den Hartog, J. E. Lawler, and M. P. Wood, Astrophys. J. Suppl. Ser. 215: 7 (2014). 
58. J. E. Lawler, M. P. Wood, E. A. Den Hartog, T. Feigenson, C. Sneden, and J. J. Cowan, Astrophys. J. Suppl. Ser. 215: 20 (2014).

59. C. E. Holmes, J. C. Pickering, M. P. Ruffoni, R. Blackwell-Whitehead, H. Nilsson, L. Engström, H. Hartman, H. Lundberg, and M. T. Belmonte, Astrophys. J. Suppl. Ser. 224: 35 (2016).

60. W. Whaling, M. T. Carle, and M. L. Pitt, J. Quant. Spectrosc. Radiat. Transfer 50, 7 (1993).

61. J. E. Lawler, C. Sneden, and J. J. Cowan, Astrophys. J. Suppl. Ser. 220: 13 (2015).

62. D. E. Nitz, S. D. Bergeson, and J. E. Lawler, J. Opt. Soc. Am. B 12, 377 (1995).

63. J. E. Lawler, C. Sneden, G. Nave, E. A. Den Hartog, N. Emrahoğlu, and J. J. Cowan, Astrophys. J. Suppl. Ser., submitted.

64. I. U. Roederer, J. E. Lawler, J. S. Sobeck, T. C. Beers, J. J. Cowan, A. Frebel, I. I. Ivans, H. Schatz, C. Sneden, and I. B. Thompson, Astrophys. J. Suppl. Ser. 203: 27 (2012).

65. J. C. Pickering, A. P. Thorne, and R. Perez, Astrophys. J. Suppl. Ser. 132, 403 (2001).

66. T. Nakamura, H. Umeda, K. Nomoto, F. K. Thielemann, and A. Burrows, Astrophys. J. 517, 193 (1999).

67. F.-K. Thielemann, K. Nomoto, and M. A. Hashimoto, Astrophys. J. 460, 408 (1996). 


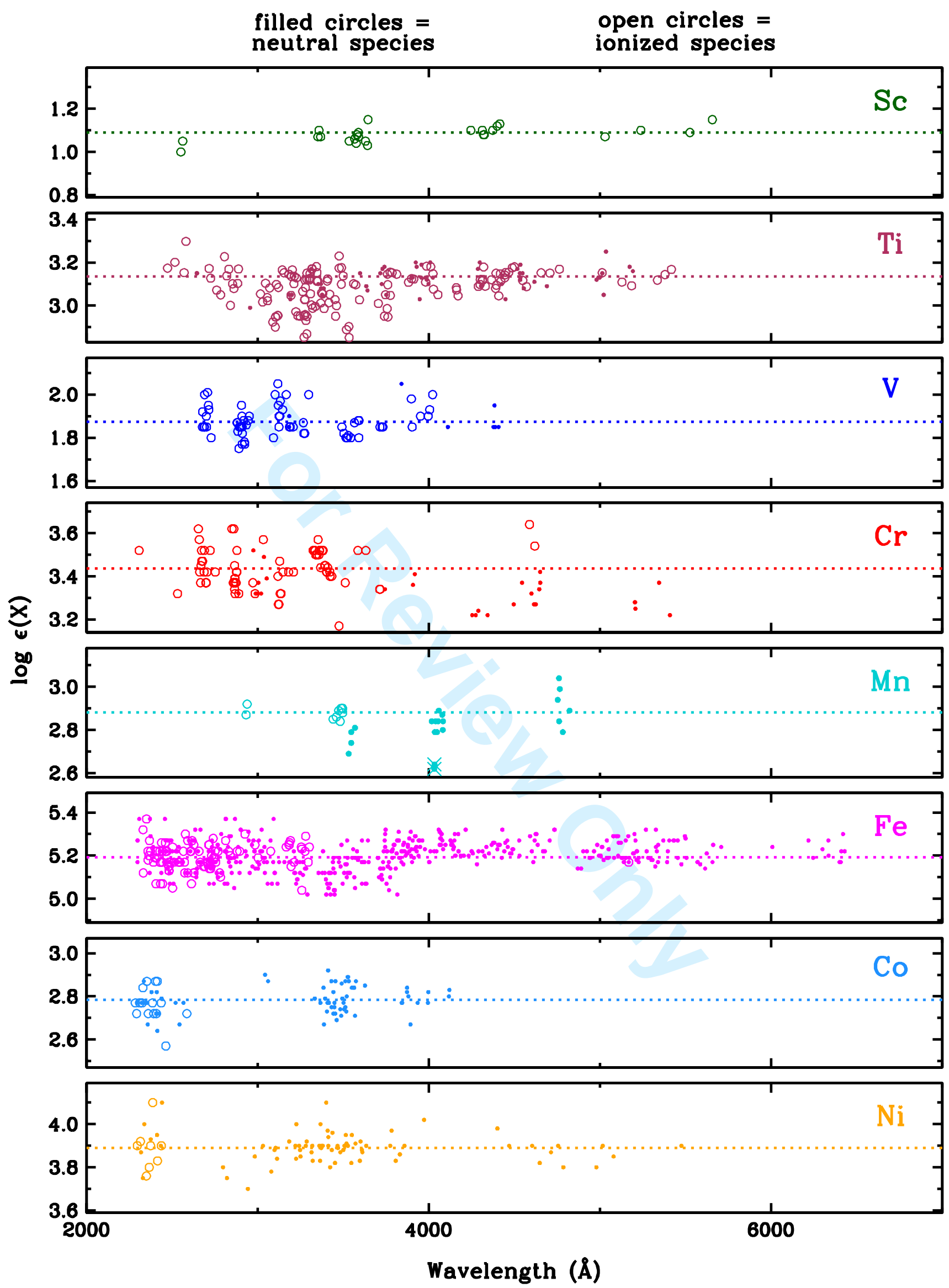

https://mc06.manuscriptcentral.com/cjp-pubs 


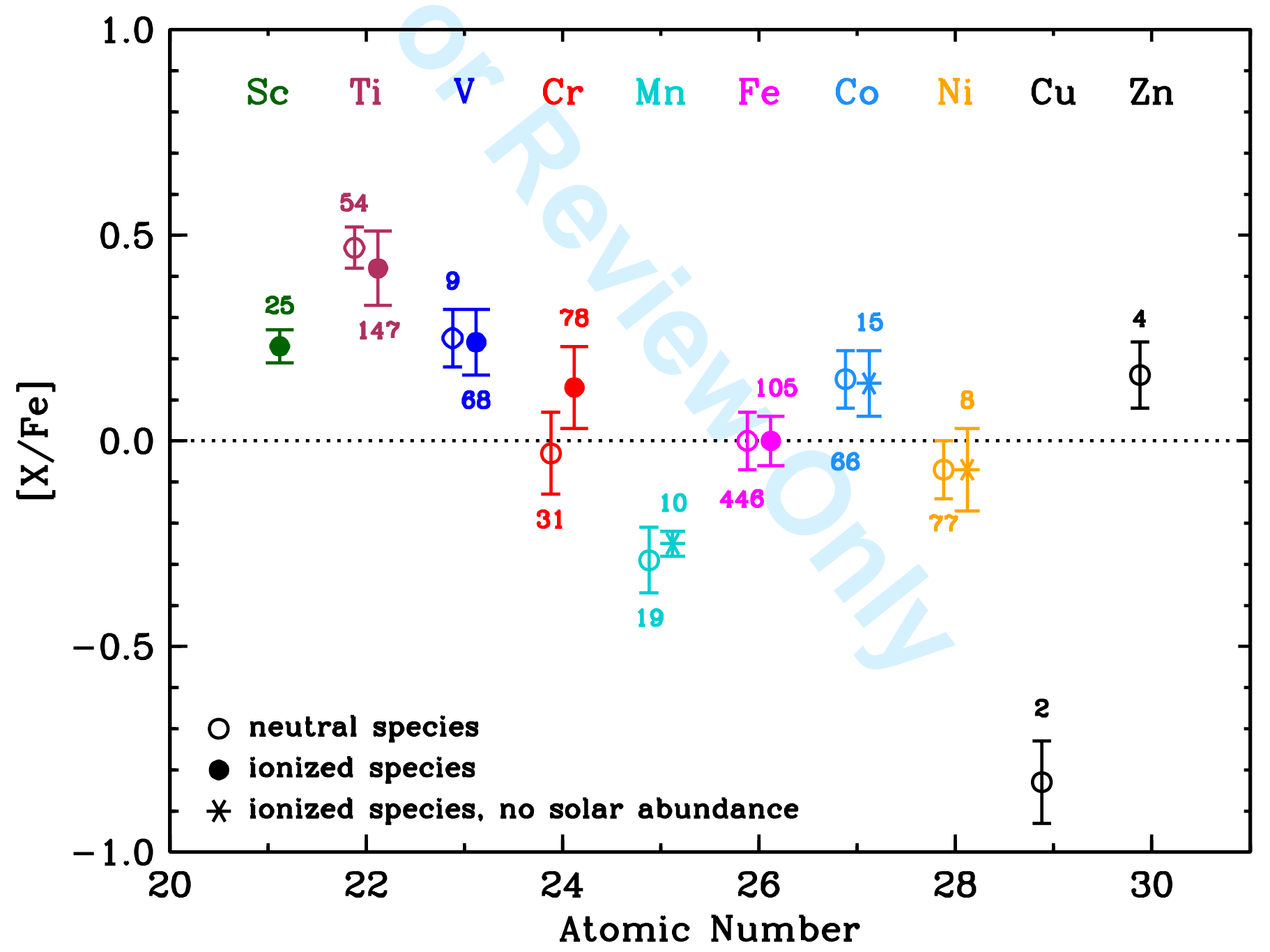

